\title{
LincRNA-p21 Inhibits the Wnt/ $\beta$-Catenin Pathway in Activated Hepatic Stellate Cells via Sponging MicroRNA-17-5p
}

\author{
Fujun Yua Yong Guo ${ }^{b}$ Bicheng Chen ${ }^{c}$ Liang Shid Peihong Donge Mengtao Zhou ${ }^{f}$ \\ Jianjian Zheng ${ }^{c}$
}

\begin{abstract}
aDepartment of Gastroenterology, The First Affiliated Hospital of Wenzhou Medical University, Wenzhou, 'ransplantation Centre, The First Affiliated Hospital of Wenzhou Medical University, Wenzhou, 'Key Laboratory of Surgery, The First Affiliated Hospital of Wenzhou Medical University, Wenzhou, dDepartment of Laboratory Medicine, The First Affiliated Hospital of Wenzhou Medical University, Wenzhou, eDepartment of Infectious Diseases, The First Affiliated Hospital of Wenzhou

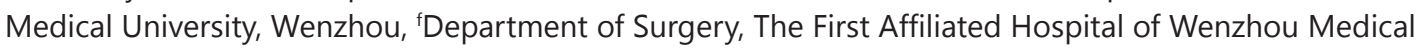
University, Wenzhou, China
\end{abstract}

\section{Key Words}

LincRNA-p21 • microRNA-17-5p • Wnt/ $\beta$-catenin pathway $\bullet$ Hepatic stellate cells $•$ Salvianolic acid $B$

\begin{abstract}
Background/Aims: It is known that the activation of hepatic stellate cells (HSCs) is a pivotal step in the initiation and progression of liver fibrosis. Aberrant activated Wnt/ $\beta$-catenin pathway is known to accelerate the development of liver fibrosis. microRNAs (miRNAs)mediated Wnt/ $\beta$-catenin pathway has been reported to be involved in HSC activation during liver fibrosis. However, whether long noncoding RNAs (IncRNAs) regulate $\mathrm{Wnt} / \beta$-catenin pathway during HSC activation still remains unclear. Methods: Long intergenic noncoding RNA-p21 (lincRNA-p21) expression was detected in Salvianolic acid B (Sal B)-treated cells. Effects of lincRNA-p21 knockdown on HSC activation and Wnt/ $\beta$-catenin pathway activity were analyzed in Sal B-treated cells. In lincRNA-p21-overexpressing cells, effects of miR-17-5p on HSC activation and Wnt/ $\beta$-catenin pathway activity were examined. Results: LincRNA-p21 expression was up-regulated in HSCs after Sal B treatment. In primary HSCs, lincRNA-p21 expression was down-regulated at Day 5 relative to Day 2. Sal B-inhibited HSC activation including the reduction of cell proliferation, $\alpha$-smooth muscle actin $(\alpha-S M A)$ and type I collagen was inhibited by lincRNA-p21 knockdown. Sal B-induced Wnt/ $\beta$-catenin pathway inactivation was blocked down by loss of lincRNA-p21. Notably, lincRNA-p21, confirmed as a target of miR-17-5p, suppresses miR-17-5p level. Lack of the miR-17-5p binding site in lincRNA-p21 prevents the suppression of miR-17-5p expression. In addition, the suppression of HSC activation and Wnt/ $\beta$-catenin pathway induced by lincRNA-p21 overexpression was almost inhibited by miR-17-5p. Conclusion: We demonstrate that lincRNA-p21-inhibited

P. Dong, M. Zhou and J. Zheng contributed equally to this work.

Jianjian Zheng, Mengtao Zhou and Peihong Dong

Key Laboratory of Surgery; Department of Surgery; Department of Infectious Diseases; The First Affiliated Hospital of Wenzhou Medical University, No.2 fuxue lane, Wenzhou, Zhejiang (P.R. China)

E-Mail 120378196@qq.com / zmt0417@hotmail.com / dongpeihong111@163.com
\end{abstract}

KARGER 
Wnt/B-catenin pathway is involved in the effects of Sal B on HSC activation and lincRNA-p21 suppresses HSC activation, at least in part, via miR-17-5p-mediated-Wnt/ $\beta$-catenin pathway.

\section{Introduction}

Liver fibrosis, representing a wound-healing response of the liver to infectious, toxic or metabolic agents, is the final common pathway of chronic liver diseases [1]. In particularly, liver fibrosis is a major cause of morbidity and mortality worldwide, leading to a big medical problem. Liver fibrosis is characterized by an abundant deposition of extracellular matrix (ECM) proteins such as collagens, associated with the distortion of normal liver architecture. Advanced liver fibrosis may finally result in cirrhosis and hepatocellular carcinoma [2]. In liver fibrosis, it is well known that activated hepatic stellate cells (HSCs) are considered as the principal cell type to promote the synthesis and deposition of ECM proteins [3]. Thus, effectively inhibiting the activation of HSC is a good therapeutic strategy for the treatment of liver fibrosis.

Regulatory noncoding RNAs (ncRNAs), such as microRNAs (miRNAs), have been reported to serve as negative regulators of gene expression by repressing protein translation or causing mRNA degradation [4]. It is well known that miRNAs take part in the control of diverse biological processes, including proliferation, differentiation, and apoptosis $[5,6]$. Numerous studies have demonstrated that miRNAs are involved in the regulation of HSC activation[7-9]. For example, loss of miR-33a expression inhibits HSC activation and ECM protein production, at least in part, via the activation of PI3K/Akt pathway and PPAR- $\alpha$ [10]. Other ncRNAs, such as long intergenic noncoding RNAs (lincRNAs) and the heterogeneous group of long noncoding RNAs (lncRNAs), have been demonstrated to be deregulated in many human diseases including liver fibrosis $[11,12]$. LncRNAs structurally resemble mRNAs but do not encode proteins, and is characterized by longer than 200 nucleotides in molecule length. Deregulated IncRNAs are reported to be involved in the regulation of HSC activation. For example, we previously found that long intergenic noncoding RNA-p21 (lincRNA-p21) suppresses activated HSCs through p21 [13]. However, the underlying mechanism of the role of lncRNA-p21 in liver fibrosis is still not completely understood.

Salvianolic acid B (Sal B) (i.e., lithospermates B, $\mathrm{C}_{36} \mathrm{H}_{30} \mathrm{O}_{16}$, molecular weight $=718 \mathrm{~g}$ ) is one of water soluble component extracted from Radix Salviae miltiorrhizae, which is a traditional herb in China. Sal B has been demonstrated to suppress liver fibrosis in animal model as well as patients with chronic hepatitis B $[14,15]$. But so far, the role of IncRNA in the anti-fibrotic effects of Sal B on liver fibrosis had never been studied.

\section{Materials and Methods}

\section{Materials}

Sal B (purity 99\%) was extracted and identified by the Chinese National Institute for the Control of Pharmaceutical and Biological Products (Beijing, China). Antibodies against type I collagen, $\alpha$-smooth muscle actin ( $\alpha$-SMA), phosphorylated $\beta$-catenin (Y86), $\beta$-catenin and $\beta$-actin were obtained from Abcam (Cambridge, MA, USA). Chemically synthesized RNAs including negative control (miR-NC) and miR-17-5p mimics were obtained from GenePharma (Shanghai, China). For transfection, the cells were transfected with $1 \mu \mathrm{g}$ of the chemically synthesized RNA.

\section{Plasmid transfection}

LincRNA-p21 siRNA (si-lnc-p21, AAGAAGAACGAGCAATTATGA), glycogen synthase kinase $3 \beta$ (GSK3 $\beta$ ) siRNA (AAGCGCTTCTCAGATAATTGC) and adenoviral vectors expressing lincRNA-p21 (Ad-lnc-p21) were designed and synthesized by Gene Pharma. The scrambled siRNA (siCtrl) and adenoviral vectors expressing 


\section{Cellular Physiology Cell Physiol Biochem 2017;41:1970-1980 \\ \begin{tabular}{l|l|l} 
and Biochemistry & $\begin{array}{l}\text { DOI: 10.1159/000472410 } \\
\text { Published onlIne: April07, } 2017\end{array}$ & $\begin{array}{l}\text { C) } 2017 \text { The Author(s). Published by S. Karger AG, Basel } \\
\text { www.karger.com/cpb }\end{array}$
\end{tabular}}

Yu et al.: LincRNA-p21 Suppresses HSC Activation through MiR-17-5p-Mediated-Wnt/BCatenin Pathway

a control scrambled sequence (Ad-Ctrl) were used as the negative control, respectively. Primary HSCs were transfected with siRNA using Lipofectamine 2000 (Invitrogen) at a final concentration of $100 \mathrm{nM}$.

Isolation and culture of mouse HSCs

Primary HSCs were isolated from male C57BL/6J mice as described previously [16]. Mice were provided by the Experimental Animal Center of Wenzhou Medical University and the experimental protocol was approved by the Institutional Animal Committee of Wenzhou Medical College. The isolated cells were cultured in Dulbecco's modified Eagle's medium with 10\% fetal bovine serum, $100 \mathrm{U} / \mathrm{mL}$ penicillin, and 100 $\mu \mathrm{g} / \mathrm{mL}$ streptomycin. The purity of cultures was confirmed by immunocytochemical staining for $\alpha$-SMA and the purity reached $>98 \%$. The primary HSCs were studied at day 2 after isolation throughout all the studies. Cells were treated with $10 \mu \mathrm{mol} / \mathrm{L}$ Sal B for $48 \mathrm{~h}$ and then transfected with si-lnc-p21 for additional $48 \mathrm{~h}$. In addition, HSCs were transduced with Ad-lnc-p21 for $48 \mathrm{~h}$ and transfected with miR-17-5p mimics for additional $48 \mathrm{~h}$. Cells were also transfected with GSK3 $\beta$ siRNA for $48 \mathrm{~h}$.

\section{Quantitative real-time PCR}

The miRNeasy Mini kit (Qiagen, Valencia, CA, USA) was used to extract total RNA in primary HSCs [6]. Fifty nanograms of total RNA was reverse-transcribed to cDNA using the ReverTra Ace qPCR RT Kit (Toyobo, Osaka, Japan). Gene expression was measured by real-time PCR using SYBR Green real-time PCR Master Mix (Toyobo, Osaka, Japan). The primers of H19, lincRNA-p21, metastasis-associated lung adenocarcinoma transcript 1 (MALAT1), Alu-mediated p21 transcriptional regulator (APTR), growth arrest-specific transcript 5 (GAS5), alpha-1 (I) collagen (Col1A1), $\alpha$-SMA, GAPDH, and U6 were designed as described previously [1722]. miR-17-5p expression was detected using TaqMan MicroRNA Assay (Applied Biosystems, Foster City, CA). The GAPDH (Applied Biosystems, Foster City, CA) level was used to normalize the relative abundance of IncRNAs and mRNAs. U6 snRNA (Applied Biosystems, Foster City, CA) was used to normalize the relative abundance of miRNAs.

\section{Western blot analysis}

To obtain total protein, HSCs were lysed with ice-cold lysis buffer (50 mM Tris-HCl, pH 7.4, $100 \mathrm{mM}$ 2 -Mercaptoethanol, $2 \% \mathrm{w} / \mathrm{v}$ SDS, $10 \%$ glycerol). Cytoplasmic and nuclear expression of $\beta$-catenin was also evaluated by western blot. Cytoplasmic and nuclear extracts were prepared according to the manufacturer's instruction (Beyotime Biotechnology, Jiangsu, China) [23, 24]. Protein samples were quantified and separated by SDS-PAGE. Then, western blot assay was performed as described previously [25].

\section{Proliferation assay}

Cells were seeded in 96-well plates at a density of $1 \times 10^{3}$ cells per well and cultured for $24 \mathrm{~h}$. Cells were ready for assessing cell proliferation after their relative treatment. Cell proliferation was assessed using CCK-8 (Dojindo, Kumamoto, Japan) according to manufacturer's instructions. Absorbance was determined at $450 \mathrm{~nm}$ on a microplate reader (Molecular Devices, Sunnyvale, CA, USA).

\section{TCF reporter activity assay}

Cells were transiently transfected with TOPFLASH and FOPFLASH (Upstate Biotechnology Inc., Lake Placid, NY, USA) using Lipofectamine 2000. Twenty-four hours after transfection, the cells were harvested and luciferase and Renilla luminescence were measured using the Dual-Luciferase Reporter Assay System (Promega, Wisconsin, WI, USA) on a luminometer (BioTek Instruments, Winooski, VT, USA). TCF reporter activity was presented as the ratio of firefly-to-Renilla luciferase activity.

RNA binding protein immunoprecipitation (RIP) assay

RIP experiment was conducted using the EZ-Magna RIP Kit (Millipore) according to the manufacturer's instructions. Briefly, primary HSCs at 80-90\% confluency were lysed in complete RIP lysis buffer, following by incubation with RIP buffer including magnetic beads coupled with anti-Argonaute-2 (Ago2) antibody (Abcam). Isotype-matched immunoglobulin G (IgG) was used as a negative control. After samples were incubated with proteinase $\mathrm{k}$, immunoprecipitated RNA was isolated. qRT-PCR was performed to analyze the levels of lincRNA-p21 and miR-17-5p in the precipitates. 


\section{Luciferase activity assay}

According to RNA22 analysis, oligonucleotides containing mouse lincRNA-p21 target sequence were annealed and cloned into the pmirGLO plasmids (Promega, Madison, WI, USA) to generate the pmioGLO-lincRNA-p21 vector: lincRNA-p21 for miR-17-5p (position of 89-113) forward, 5'-ATAGCCACAACTCTCTGCCG-3', and reverse, 5'-GCGATAGACCCGCACATACA-3'. Empty plasmid pmirGLO was used as a negative control. Luciferase reporter plasmids plus miR-17-5p mimics or miR-NC were cotransfected into HEK293T cells using Lipofectamine 2000. Forty-eight hours after transfection, relative luciferase activity was examined in a luminometer using a Dual-Luciferase Reporter Assay System (Promega).

\section{Statistical analysis}

Data from at least three independent experiments were expressed as the mean \pm SD. Comparisons between two groups and multiple groups were made using Student's $t$-test and one-way analysis of variance, respectively. $P<0.05$ was considered significant. All statistical analyses were performed with SPSS software (version 13; SPSS, Chicago, IL).

\section{Results}

LincRNA-p21 is increased by Sal B and reduced in activated primary HSCs

Recent studies have shown that many lncRNAs including H19 [12], lincRNA-p21 [13], GAS5 [19], MALAT1 [20] and APTR [21], are involved in the progression of liver fibrosis. To investigate whether these lncRNAs play a role in the effects of Sal B on HSC activation, the expressions of H19, lincRNA-p21, MALAT1, APTR and GAS5 were examined in Sal B-treated primary HSCs. Our results showed that Sal B caused an increase in lincRNA-p21 and GAS5, with no effect on H19, MALAT1 and APTR (Fig.1A). Our data indicated that the expressions of lincRNA-p21 and GAS5 were increased by 12.6-fold and 1.4-fold, respectively, in Sal B group relative to the control group (Fig.1A). Expression of lincRNA-p21 enhanced by Sal B was far higher than that of GAS5, therefore, lincRNA-p21 was chosen for the next experiment. LincRNA-p21 was also examined in primary HSCs. Compared with Day 2, lincRNA-p21 was decreased by $52 \%$ at Day 5 (Fig.1B), suggesting that lincRNA-p21 was reduced during HSC activation. These data demonstrate that lincRNA-p21, down-regulated in activated HSCs, may be involved in the effects of Sal B on HSC activation.

The inhibitory effects of Sal B on HSC activation are suppressed by the loss of lincRNA-p21

The effects of Sal B on cell proliferation, collagen expression and HSC transdifferentiation were examined in primary HSCs. As shown by CCK-8 assays, the growth rate was reduced to

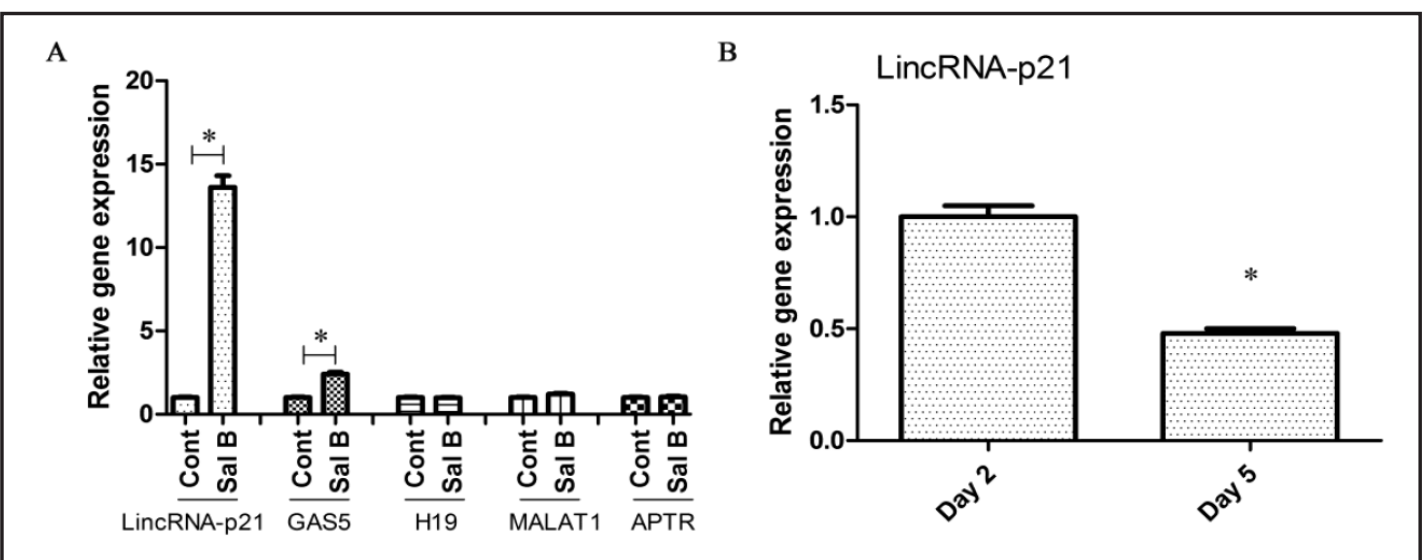

Fig. 1. Expressions of lncRNAs were examined in primary HSCs after Sal B treatment and lincRNA-p21 was reduced in activated HSCs. (A) Expressions of lncRNAs including lincRNA-p21, GAS5, H19, MALAT1 and APTR were examined in Sal B-treated primary HSCs. (B) LincRNA-p21 expression was examined at Day 2 and Day 5 . Each value is the mean \pm SD of three experiments. ${ }^{*} P<0.05$ compared with the control. 


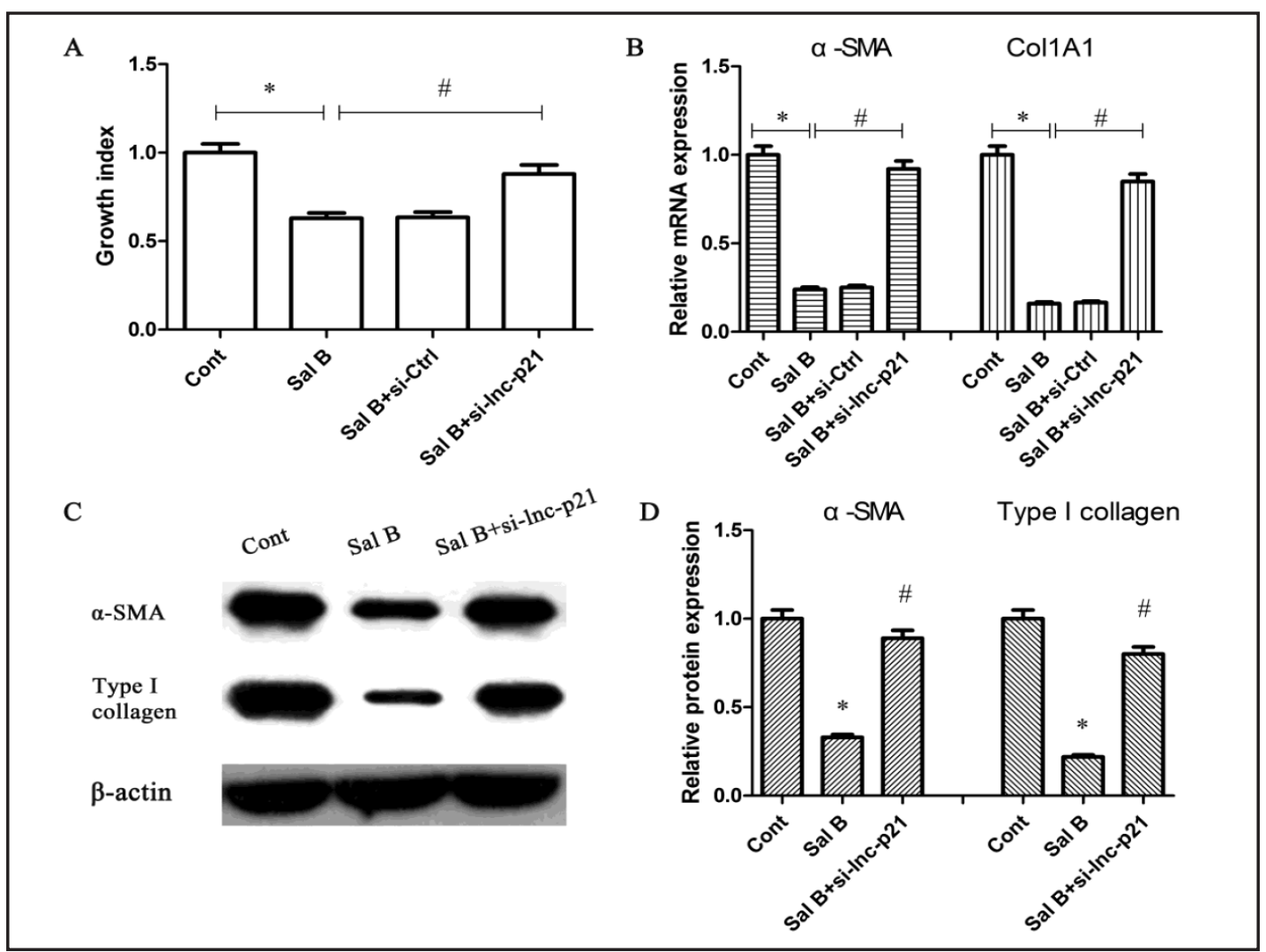

Fig. 2. LincRNA-p21 was involved in the anti-fibrotic effects induced by Sal B. Primary 2-day-old HSCs were treated with Sal B for $48 \mathrm{~h}$ and then transfected with si-lnc-p21 for $48 \mathrm{~h}$. Sal B inhibited cell proliferation (A), mRNA (B) and protein (C and D) expressions of $\alpha$-SMA and Col1A1, which was restored by loss of lincRNA-p21. Each value is the mean \pm SD of three experiments. ${ }^{*} P<0.05$ compared with the control and ${ }^{\#} P<0.05$ compared with the Sal B group.

63\% in Sal B-treated cells compared to untreated cells (Fig.2A). The results of real-time PCR indicated that the mRNA expressions of $\alpha$-SMA and Col1A1 were decreased by $76 \%$ and 84\%, respectively, in Sal B-treated cells relative to untreated cells (Fig.2B). Of note, si-Ctrl had no effect on the results caused by Sal B (Fig.2A and Fig.2B). Consistent with the mRNA results, Sal B resulted in a significant reduction in $\alpha$-SMA and type I collagen (Fig.2C and Fig.2D). Our results suggest an inhibitory role of Sal B in the activation of HSC. Next, the role of lincRNA-p21 in anti-fibrotic effects Sal B on HSC activation was examined. Interestingly, Sal B-suppressed cell proliferation was restored by lincRNA-p21 knockdown (Fig.2A). Moreover, the reduced mRNA and protein expressions of $\alpha$-SMA and Col1A1 induced by Sal B were blocked down by loss of lincRNA-p21 (Fig.2B-D). These data suggest that lincRNA-p21 plays a vital role in the effects of Sal B on HSC activation.

\section{Sal B-inhibited Wnt/ $\beta$-catenin pathway was re-activated by loss of lincRNA-p21}

Aberrant activated $\mathrm{Wnt} / \beta$-catenin pathway has been reported to contribute to the progression of liver fibrosis [25]. As shown in Fig.3A, there was a significant increase in $\beta$-catenin in cytoplasm and nucleus during culture days. In addition, c-myc, one of the downstream target genes of Wnt/ $\beta$-catenin pathway, was found to be increased during culture days (Fig.3A). Then, we examined whether Sal B had an effect on the activity of Wnt/ $\beta$-catenin pathway. Sal B treatment led to a significant reduction in TCF activity (Fig.3B). As shown by the results of western blot, the protein level of P- $\beta$-catenin was increased by 4.5 fold in Sal B-treated cells compared with untreated cells (Fig.3C). In line with it, $\beta$-catenin in cytoplasm and nucleus was significant reduced by Sal B (Fig.3C). Next, we examined the 


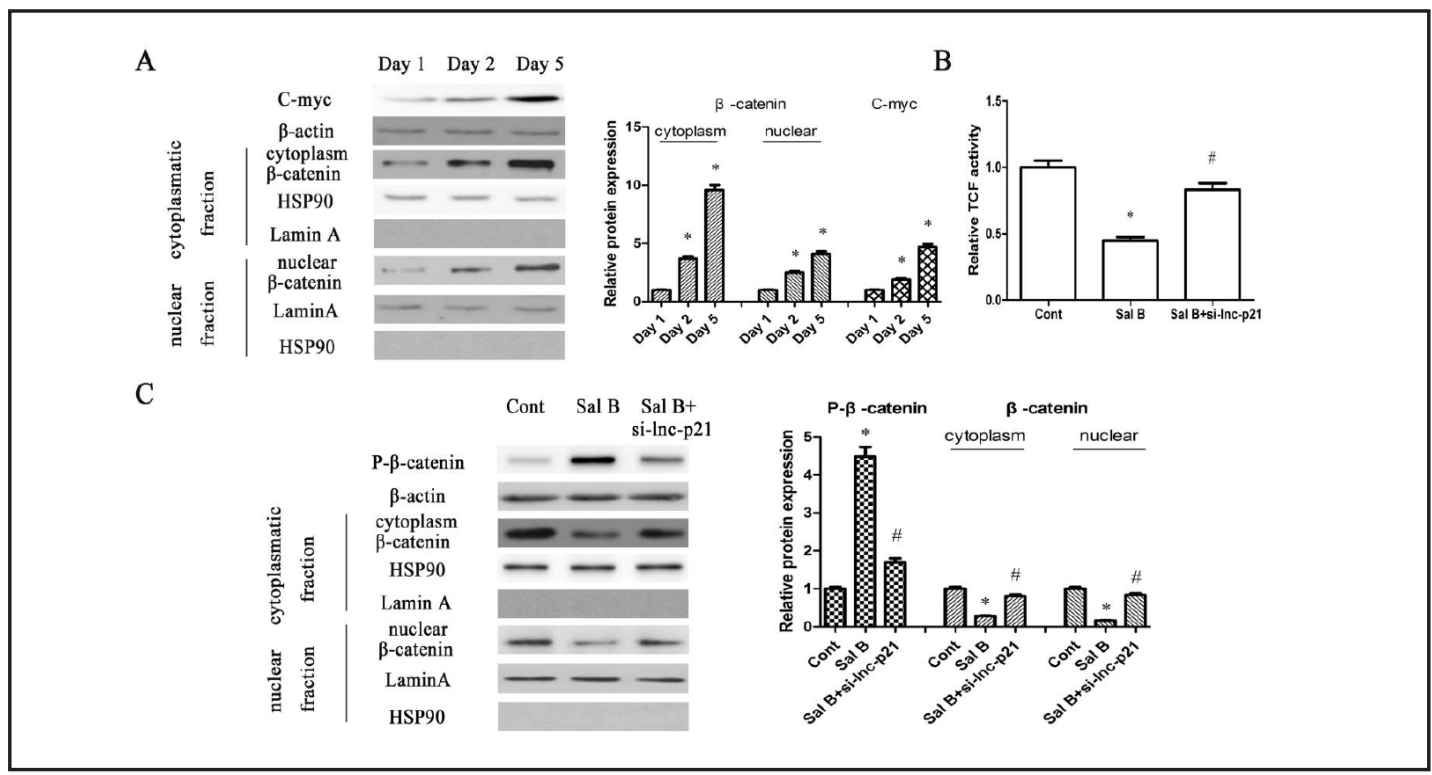

Fig. 3. Sal B-inhibited Wnt/ $\beta$-catenin pathway was blocked down by loss of lincRNA-p21. Primary 2-day-old HSCs were treated with Sal B for $48 \mathrm{~h}$ and then transfected with si-lnc-p21 for $48 \mathrm{~h}$. (A) $\beta$-catenin in cytoplasm and nuclear, and c-myc were examined in primary HSCs at Day 1, Day 2 and Day5. (B) TCF activity. (C) P- $\beta$-catenin and $\beta$-catenin in cytoplasm and nuclear. Each value is the mean \pm SD of three experiments. ${ }^{*} P<0.05$ compared with the control and ${ }^{\#} P<0.05$ compared with the Sal B group.

Fig. 4. Interaction of miR-17-5p with lincRNA-p21. (A) miR-17-5p was detected in cells transduced with Ad-lnc-p21 or Ad-lnc-p21-mut. (B) Schematic diagram of the miR-17-5p binding site in the lincRNA-p21 based on RNA22 software. (C) Relative luciferase activities of luciferase reporters bearing wild-type or mutant lincRNA-p21 were analyzed $48 \mathrm{~h}$ following transfection with the indicated miR-17-5p mimics or miR-NC in HEK293T cells. ${ }^{*} P<0.05$ compared with the control.

activity of Wnt/ $\beta$-catenin pathway in Sal B-treated cells transfected with si-lnc-p21. Of note, lincRNA-p21 knockdown led to the restoration of Sal B-inhibited TCF activity (Fig.3B). Loss of lincRNA-p21 inhibited Sal B-induced P- $\beta$-catenin (Fig.3C). Meanwhile, $\beta$-catenin protein reduced by Sal B in cytoplasm and nucleus was restored by loss of lincRNA-p21 (Fig.3C). Our data demonstrate that Sal B inhibited Wnt/ $\beta$ catenin pathway was through lincRNA-p21. To exclude nuclear contamination in the cytoplasmatic fraction, LaminA (a marker for the nuclear fraction) was detected in the cytoplasmatic fraction. Also, HSP90 (a marker for the cytoplasmatic fraction) was detected in the nuclear fraction. As shown

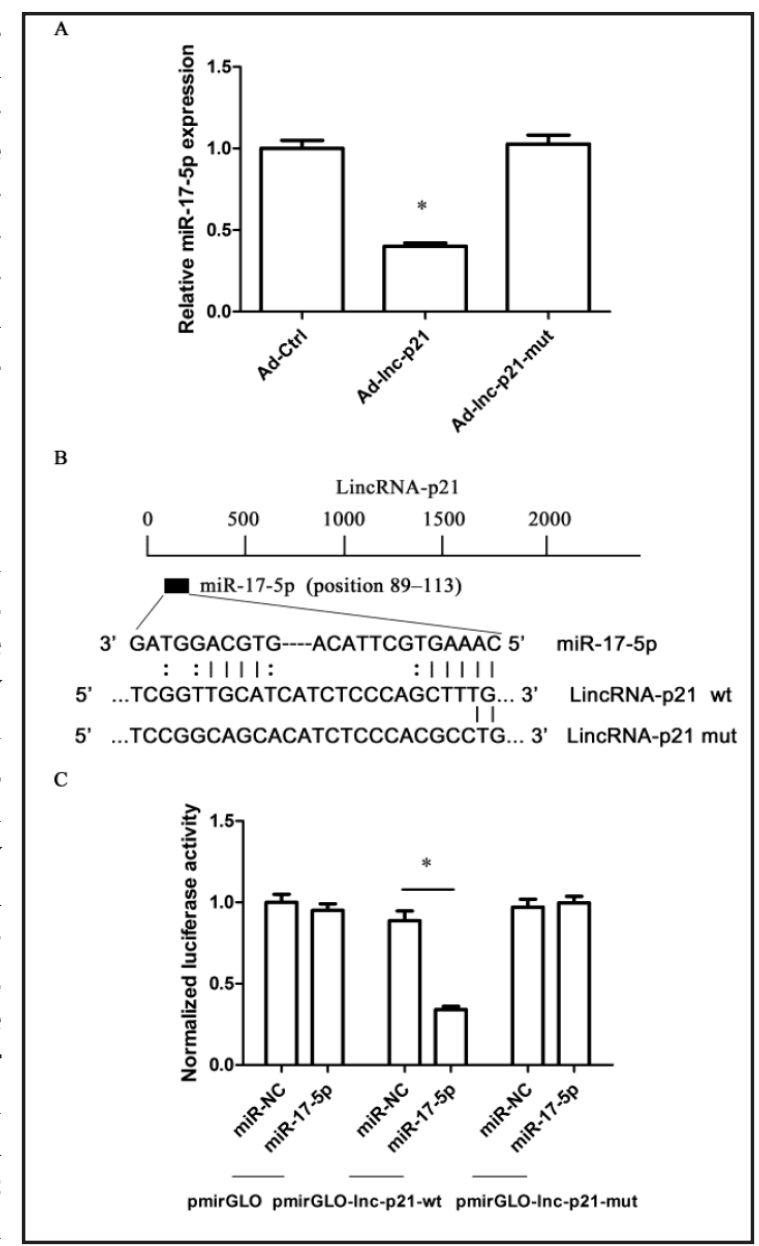


A

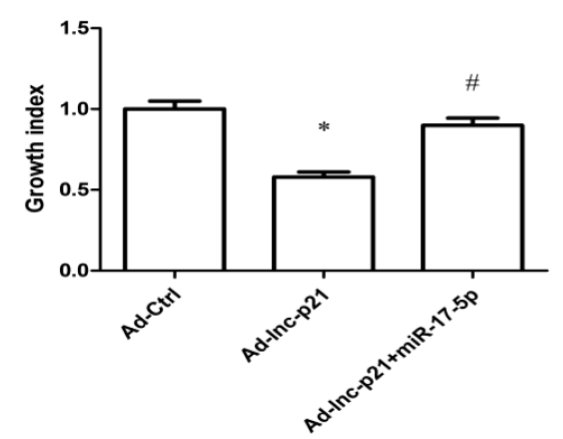

$\mathrm{C}$

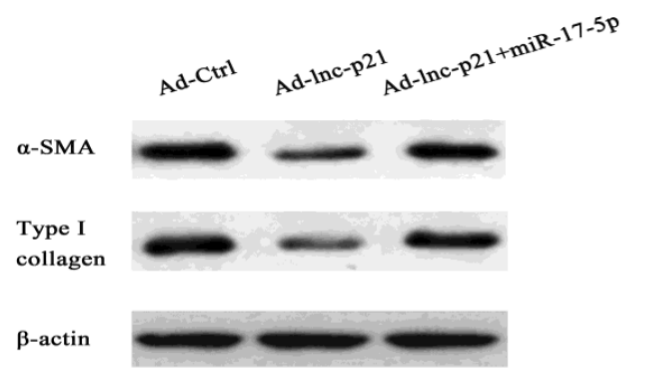

B

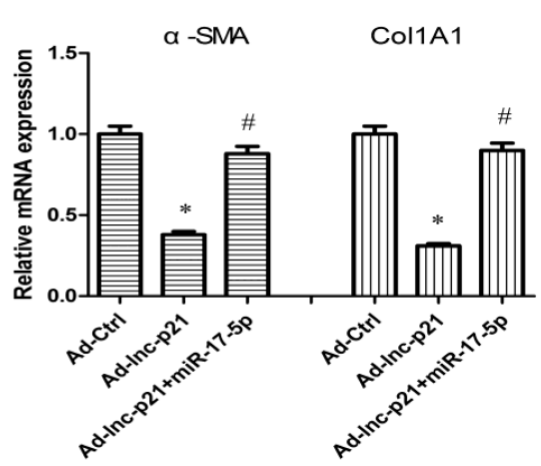

D

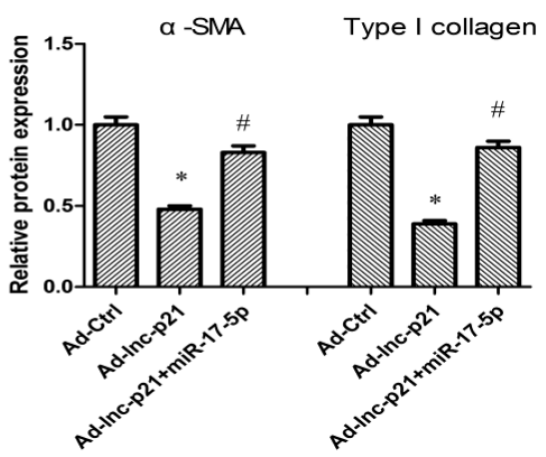

Fig. 5. LincRNA-p21-inhibited HSC activation was blocked down by miR-17-5p. Primary 2-day-old HSCs were treated with Ad-Inc-p21 for $48 \mathrm{~h}$ and then transfected with miR-17-5p for $48 \mathrm{~h}$. LincRNA-p21 suppressed cell proliferation (A), mRNA (B) and protein (C and D) expressions of $\alpha$-SMA and Col1A1, which was restored by miR-17-5p. Each value is the mean \pm SD of three experiments. ${ }^{*} P<0.05$ compared with the control and ${ }^{\#} P<0.05$ compared with the Ad-lnc-p21 group.

in Fig.3A and Fig.3C, LaminA was not found in the cytoplasmatic fraction and HSP90 not found in the nuclear fraction.

LincRNA-p21 inactivates Wnt/ $\beta$-catenin pathway was through miR-17-5p

Our previous study has demonstrated that miR-17-5p overexpression contributes to the activation of Wnt/ $\beta$-catenin pathway and Sal B inhibited Wnt/ $\beta$-catenin pathway via miR17-5p [25]. To determine whether miR-17-5p is involved in lincRNA-p21-inhibited Wnt/ $\beta$ catenin pathway, miR-17-5p was detected in lincRNA-p21-overexpressing HSCs. There was a significant reduction in miR-17-5p in lincRNA-p21-overexpressing HSCs (Fig.4A). Bioinformatic analysis (RNA22) for miRNA recognition sequences on lincRNA-p21 revealed the presence of a putative miR-17-5p site (Fig.4B). To investigate whether lincRNA-p21 is a target of miR-17-5p, we used pmirGLO construct to generate a lincRNA-p21 luciferase reporter containing the miR-17-5p-binding sites (pmirGLO-lnc-p21-wt) or mutated sites (pmirGLO-lnc-p21-mut). miR-17-5p caused a reduction in luciferase activity of pmirGLOlnc-p21-wt (Fig.4C). Moreover, miR-17-5p had no effect on luciferase activity of pmirGLOlnc-p21-mut (Fig.4C). In line with it, lincRNA-p21 overexpression with the mutation of the miR-17-5p binding site did not suppress miR-17-5p expression, indicating that lack of the miR-17-5p binding site in lincRNA-p21 prevents the suppression of miR-17-5p expression (Fig.4A). Taken together, lincRNA-p21 is a target of miR-17-5p.

miR-17-5p is involved in the effects of lincRNA-p21 on HSC activation

To investigate the role of miR-17-5p in the effects of lincRNA-p21 on HSC activation, cell proliferation, collagen expression and HSC transdifferentiation were examined in lincRNA-p21-overexpressing cells transfected with miR-17-5p mimics. Our results showed 


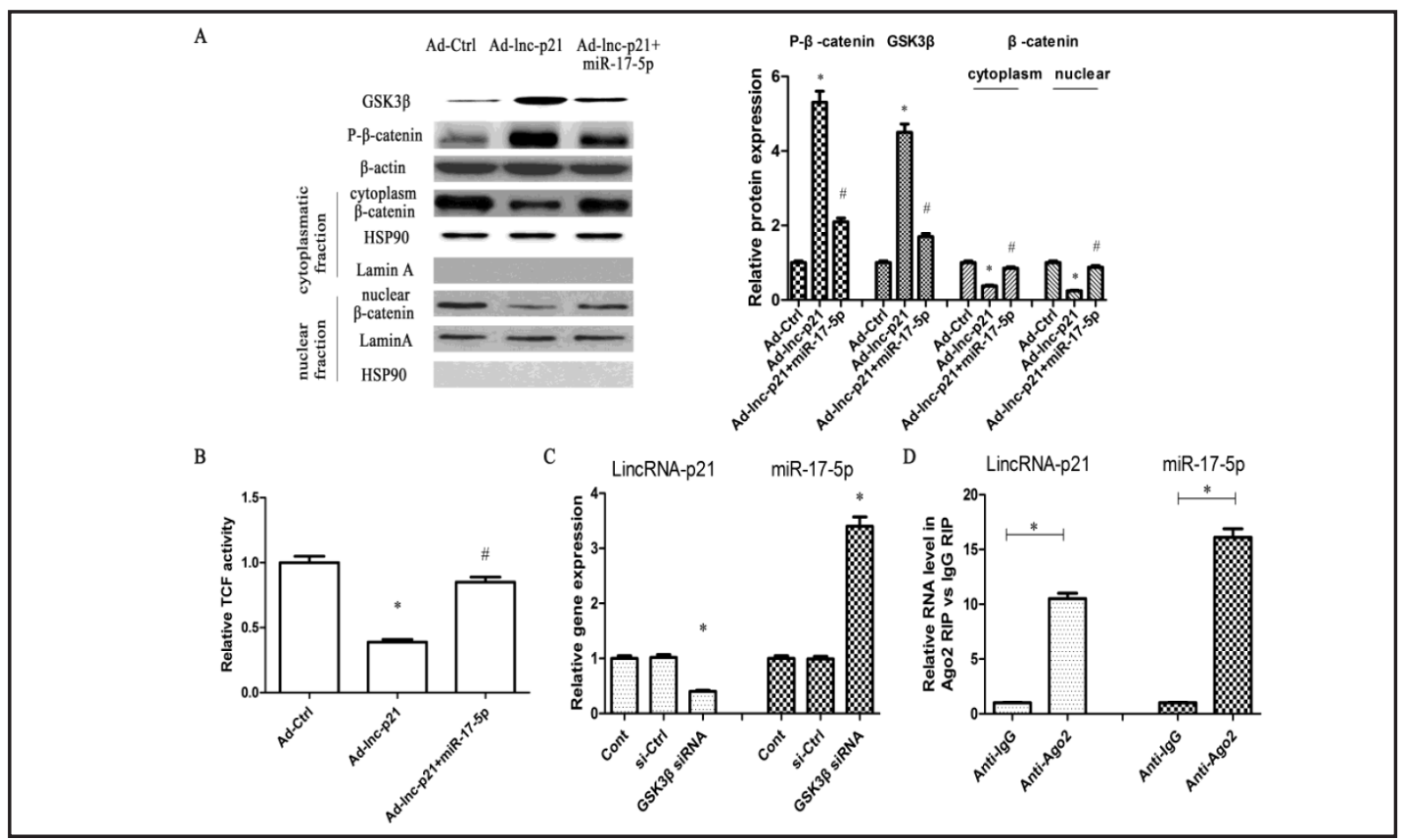

Fig. 6. LincRNA-p21-inhibited Wnt/ $\beta$-catenin pathway was re-activated by miR-17-5p. Primary 2-day-old HSCs were treated with Ad-lnc-p21 for $48 \mathrm{~h}$ and then transfected with miR-17-5p for $48 \mathrm{~h}$. (A) The protein levels of P- $\beta$-catenin, GSK3 $\beta$ and $\beta$-catenin in cytoplasm and nuclear. (B) TCF activity. (C) The effects of loss of GSK3 $\beta$ on lincRNA-p21 and miR-17-5p. (D) Association of lincRNA-p21 and miR-17-5p with Ago2 was detected by RIP experiment. Relative levels of lincRNA-p21 and miR-17-5p were expressed as fold enrichment in Ago2 relative to IgG immunoprecipitates by qRT-PCR. ${ }^{*} P<0.05$ compared with the control and ${ }^{\#} P<0.05$ compared with the Ad-lnc-p21 group.

that lincRNA-p21 overexpression led to a reduction in cell growth rate (Fig.5A). Also, the expressions of type I collagen and $\alpha$-SMA were down-regulated by lincRNA-p21 (Fig.5B-D). These results confirm the inhibitory role of lincRNA-p21 in HSC activation. However, these effects induced by lincRNA-p21 were almost blocked down by miR-17-5p, suggesting that lincRNA-p21 inhibited HSC activation, at least in part, via miR-17-5p (Fig.5).

\section{miR-17-5p inhibited lincRNA-p21-induced Wnt/ $\beta$-catenin pathway inactivation}

To further confirm whether lincRNA-p21 inhibits miR-17-5p-mediated Wnt/ $\beta$ catenin pathway, we examined P- $\beta$-catenin, $\beta$-catenin and TCF activity in lincRNA-p21overexpression cells transfected with miR-17-5p. As expected, increased P- $\beta$-catenin and reduced TCF activity induced by lincRNA-p21 were inhibited by miR-17-5p (Fig.6A and Fig.6B). In line with it, miR-17-5p restored lincRNA-p21-suppressed $\beta$-catenin in cytoplasm and nucleus (Fig.6A). Notably, as shown in Fig.6A, there was no LaminA in the cytoplasmatic fraction and no HSP90 in the nuclear fraction. In addition, the protein expression of GSK3 $\beta$, a key element of $\beta$-catenin/Wnt pathway, was examined in lincRNA-p21-overexpression cells transfected with miR-17-5p. It was found that GSK3 $\beta$ was increased by lincRNA-p21, which was blocked down by miR-17-5p (Fig.6A). Interestingly, the loss of GSK3 $\beta$ led to an increase in miR-17-5p and a reduction in lincRNA-p21 (Fig.6C), suggesting that activated Wnt/ $\beta$ catenin pathway inhibited lincRNA-p21 and contributed to enhance miR-17-5p expression. Of note, RIP experiment showed that compared with IgG pellets, lincRNA-p21 and miR17-5p were enriched by 10.5-fold and 16.1-fold in the Ago2 pellet, respectively (Fig.6D). In line with luciferase assays, our data confirmed that lincRNA-p21 is recruited to Ago2related RNA-induced silencing complexes and functionally interacts with miR-17-5p. Our results demonstrate that lincRNA-p21 induces the inactivation of Wnt/ $\beta$-catenin pathway is through miR-17-5p. 


\section{Discussion}

It is known that there is an increase in cell proliferation, ECM production and $\alpha$-SMA synthesis in activated HSCs compared with quiescent HSCs [26]. In our study, Sal B treatment caused the suppression of activated HSCs including the reduction of cell growth rate, type I collagen and $\alpha$-SMA. Interestingly, it was found that lincRNA-p 21 was increased by Sal B. Loss of lincRNA-p21 resulted in the suppression of Sal B-inhibited HSC activation, indicating that lincRNA-p21 may be responsible for the effects of Sal B on HSC activation. Further studies showed that the inactivation of Wnt/ $\beta$-catenin pathway induced by Sal B was re-activated by lincRNA-p21 knockdown. Our data demonstrate that Sal B inhibits HSC activation, at least in part, through lincRNA-p21- mediated $\mathrm{Wnt} / \beta$-catenin pathway and this is a first report.

Sustained activated Wnt/ $\beta$-catenin pathway is involved in the activation of HSCs $[27,28]$. In this study, as indicated by the results of western blot, $\beta$-catenin in cytoplasm and nucleus was increased during HSC activation. By contrast, inactivated Wnt/ $\beta$-catenin pathway contributes to the suppression of liver fibrosis. For example, the activation of HSCs could be inhibited by Wnt antagonist such as Dickkopf-1 (Dkk-1) in vitro [27, 29]. miR-17$5 p$, often deregulated in various cancers, has been reported to act as an oncogenic miRNA [30-32]. Previously, we demonstrated that miRNA-17-5p-activated Wnt/ $\beta$-catenin pathway contributes to the progression of liver fibrosis and is involved in the anti-fibrotic effects of Sal B on HSC activation [25]. In this study, we found that miR-17-5p was reduced by lincRNA-p21. Luciferase activity assays revealed that lincRNA-p21 is a target of miR-17-5p. Further studies showed that the effects of lincRNA-p21 on HSC activation and Wnt/ $\beta$-catenin pathway were blocked down by miR-17-5p. All these data suggest that miR-17-5p-mediated Wnt/ß-catenin pathway is involved in the effects of lincRNA-p21 on HSC activation. A recent study has been reported that non-canonical Wnt is involved in activated rat hepatic stellate cells through influencing HSC survival and paracrine stimulation of Kupffer cells [33]. It is not clear whether non-canonical Wnt is involved in the effects of lincRNA-p21 on HSC activation and further studies are warranted to prove it.

In past decades, IncRNAs are generally considered as simply transcriptional "noise" or cloning artifacts. Emerging evidence has demonstrated that lncRNAs can act as vital regulators in the control of fundamental biological processes at multiple levels [20]. LncRNAs have been reported to regulate various cellular functions through diverse molecular mechanisms such as chromatin modification, transcriptional regulation and post-transcriptional regulation [11, 34, 35]. Recently, lncRNAs have been shown to serve as competing endogenous RNAs (ceRNAs) to sponge miRNAs, consequently modulating the de-repression of miRNA targets $[36,37]$. For example, Wang et al. found that up-regulated lncRNA-UCA1 contributes to progression of hepatocellular carcinoma through inhibition of miR-216b [37]. LincRNA-p21 has been reported to be deregulated in various human diseases and act as a tumor suppressor in cancers. For instance, Wang et al. found that lincRNA-p21 suppresses $\beta$-catenin signaling and tumorigenicity of colorectal cancer stem cells [38]. In line with it, we found that lincRNA-p21 suppresses Wnt/ $\beta$-catenin signal through sponging miR-17-5p. In vitro, lincRNA-p21 contributes to a reduction in miR-17-5p. With the restoration of miR-17$5 p$, the anti-fibrotic effects of lincRNA-p21 were almost inhibited. Moreover, lincRNA-p21inhibited Wnt/ $\beta$-catenin pathway was also blocked down by miR-17-5p overexpression. Notably, lincRNA-p21 is a target of miR-17-5p and lack of the miR-17-5p binding site in lincRNA-p21 prevents the suppression of miR-17-5p expression. Consistent with luciferase assays, RIP assays further demonstrated that lincRNA-p21 is recruited to Ago2-related RNAinduced silencing complexes and functionally interacts with miR-17-5p. Our data reveal that lincRNA-p21 inhibits HSC activation, at least in part, through inhibiting miR-17-5p-mediaed Wnt/ $\beta$-catenin pathway. However, the mechanism of the direct regulation of lincRNA-p21 by Sal B still remains largely unknown and further studies are warranted.

In conclusion, our results reveal that lincRNA-p21-inhibited Wnt/ $\beta$-catenin pathway is involved in the effects of Sal B on HSC activation and lincRNA-p21 suppresses HSC activation, 


\section{Cellular Physiology Cell Physiol Biochem 2017;41:1970-1980 \\ \begin{tabular}{ll|l} 
DOI: 10.1159/000472410 & $\begin{array}{l}\text { O 2017 The Author(s). Published by S. Karger AG, Basel } \\
\text { wwww.karger.com/cpb }\end{array}$
\end{tabular} \\ Yu et al.: LincRNA-p21 Suppresses HSC Activation through MiR-17-5p-Mediated-Wnt/ß- Catenin Pathway}

at least in part, via miR-17-5p-mediated-Wnt/ $\beta$-catenin pathway. Our results provide a new insight of the role of lincRNA-p21-inhibited Wnt/ $\beta$-catenin signaling in liver fibrosis.

\section{Acknowledgements}

The project was supported by the National Natural Science Foundation of China (No. 81500458 and No. 81501823), Zhejiang Provincial Natural Science Foundation of China (LY16H030012 and LY17H200005) and Wenzhou Municipal Science and technology Bureau (Y20150091, Y20160071 and Y20160077).

\section{Disclosure Statement}

None.

\section{References}

1 Hernandez-Gea V, Friedman SL: Pathogenesis of liver fibrosis. Annu Rev Pathol 2011;6:425-456.

- Wei J, Feng L, Li Z, Xu G, Fan X: MicroRNA-21 activates hepatic stellate cells via PTEN/Akt signaling. Biomed Pharmacother 2013;67:387-392.

-3 Tu X, Zhang H, Zhang J, Zhao S, Zheng X, Zhang Z, Zhu J, Chen J, Dong L, Zang Y: MicroRNA-101 suppresses liver fibrosis by targeting the TGFbeta signalling pathway. J Pathol 2014;234:46-59.

4 Croce CM, Calin GA: miRNAs, cancer, and stem cell division. Cell 2005;122:6-7.

5 Ghildiyal M, Zamore PD: Small silencing RNAs: an expanding universe. Nat Rev Genet 2009;10:94-108.

6 Zheng J, Wu C, Lin Z, Guo Y, Shi L, Dong P, Lu Z, Gao S, Liao Y, Chen B, Yu F: Curcumin up-regulates phosphatase and tensin homologue deleted on chromosome 10 through microRNA-mediated control of DNA methylation--a novel mechanism suppressing liver fibrosis. FEBS J 2014;281:88-103.

7 Yu F, Yang J, Huang K, Pan X, Chen B, Dong P, Zheng J: The Epigenetically-Regulated microRNA-378a Targets TGF-beta2 in TGF-beta1-Treated Hepatic Stellate Cells. Cell Physiol Biochem 2016;40:183-194.

8 Yu F, Fan X, Chen B, Dong P, Zheng J: Activation of Hepatic Stellate Cells is Inhibited by microRNA-378a-3p via Wnt10a. Cell Physiol Biochem 2016;39:2409-2420.

9 Li G, Li J, Li C, Qi H, Dong P, Zheng J, Yu F: MicroRNA-125a-5p Contributes to Hepatic Stellate Cell Activation through Targeting FIH1. Cell Physiol Biochem 2016;38:1544-1552.

10 Li ZJ, Ou-Yang PH, Han XP: Profibrotic effect of miR-33a with Akt activation in hepatic stellate cells. Cell Signal 2014;26:141-148.

-11 He Y, Wu YT, Huang C, Meng XM, Ma TT, Wu BM, Xu FY, Zhang L, Lv XW, Li J: Inhibitory effects of long noncoding RNA MEG3 on hepatic stellate cells activation and liver fibrogenesis. Biochim Biophys Acta 2014;1842:2204-2215.

12 Yang JJ, Liu LP, Tao H, Hu W, Shi P, Deng ZY, Li J: MeCP2 silencing of LncRNA H19 controls hepatic stellate cell proliferation by targeting IGF1R. Toxicology 2016;359-360:39-46.

13 Zheng J, Dong P, Mao Y, Chen S, Wu X, Li G, Lu Z, Yu F: lincRNA-p21 inhibits hepatic stellate cell activation and liver fibrogenesis via p21. FEBS J 2015;282:4810-4821.

-14 Liu P, Hu YY, Liu C, Zhu DY, Xue HM, Xu ZQ Xu LM, Liu CH, Gu HT, Zhang ZQ: Clinical observation of salvianolic acid B in treatment of liver fibrosis in chronic hepatitis B. World J Gastroenterol 2002;8:679685.

15 Li S, Wang L, Yan X, Wang Q, Tao Y, Li J, Peng Y, Liu P, Liu C: Salvianolic Acid B Attenuates Rat Hepatic Fibrosis via Downregulating Angiotensin II Signaling. Evid Based Complement Alternat Med 2012;2012:160726.

16 Chang W, Yang M, Song L, Shen K, Wang H, Gao X, Li M, Niu W, Qin X: Isolation and culture of hepatic stellate cells from mouse liver. Acta Biochim Biophys Sin (Shanghai) 2014;46:291-298. 


\section{Cellular Physiology Cell Physiol Biochem 2017;41:1970-1980 \begin{tabular}{ll|l} 
DOI: 10.1159/000472410 & Ond Biochemistry 2017 The Author(s). Published by S. Karger AG, Basel \\
wwww.karger.com/cpb
\end{tabular} and Biochemistry}

Yu et al.: LincRNA-p21 Suppresses HSC Activation through MiR-17-5p-Mediated-Wnt/BCatenin Pathway

17 Wu G, Cai J, Han Y, Chen J, Huang ZP, Chen C, Cai Y, Huang H, Yang Y, Liu Y, Xu Z, He D, Zhang X, Hu X, Pinello L, Zhong D, He F, Yuan GC, Wang DZ, Zeng C: LincRNA-p21 regulates neointima formation, vascular smooth muscle cell proliferation, apoptosis, and atherosclerosis by enhancing p53 activity. Circulation 2014;130:1452-1465.

18 Zheng J, Lin Z, Dong P, Lu Z, Gao S, Chen X, Wu C, Yu F: Activation of hepatic stellate cells is suppressed by microRNA-150. Int J Mol Med 2013;32:17-24.

-19 Yu F, Zheng J, Mao Y, Dong P, Lu Z, Li G, Guo C, Liu Z, Fan X: Long Non-coding RNA Growth Arrest-specific Transcript 5 (GAS5) Inhibits Liver Fibrogenesis through a Mechanism of Competing Endogenous RNA. J Biol Chem 2015;290:28286-28298.

20 Yu F, Lu Z, Cai J, Huang K, Chen B, Li G, Dong P, Zheng J: MALAT1 functions as a competing endogenous RNA to mediate Rac1 expression by sequestering miR-101b in liver fibrosis. Cell Cycle 2015;14:3885-3896.

21 Yu F, Zheng J, Mao Y, Dong P, Li G, Lu Z, Guo C, Liu Z, Fan X: Long non-coding RNA APTR promotes the activation of hepatic stellate cells and the progression of liver fibrosis. Biochem Biophys Res Commun 2015;463:679-685.

22 Gao L, Liu Y, Wen Y, Wu W: LncRNA H19-mediated mouse cleft palate induced by all-trans retinoic acid. Hum Exp Toxicol 2016;10.1177/0960327116651121

23 Yu M, Cai L, Liang M, Huang Y, Gao H, Lu S, Fei J, Huang F: Alteration of NRSF expression exacerbating 1-methyl-4-phenyl-pyridinium ion-induced cell death of SH-SY5Y cells. Neurosci Res 2009;65:236-244.

-24 Lu J, Zhang F, Zhao D, Hong L, Min J, Zhang L, Li F, Yan Y, Li H, Ma Y, Li Q: ATRA-inhibited proliferation in glioma cells is associated with subcellular redistribution of beta-catenin via up-regulation of Axin. J Neurooncol 2008;87:271-277.

-25 Yu F, Lu Z, Huang K, Wang X, Xu Z, Chen B, Dong P, Zheng J: MicroRNA-17-5p-activated Wnt/beta-catenin pathway contributes to the progression of liver fibrosis. Oncotarget 2016;7:81-93.

-26 Lin J, Chen A: Activation of peroxisome proliferator-activated receptor-gamma by curcumin blocks the signaling pathways for PDGF and EGF in hepatic stellate cells. Lab Invest 2008;88:529-540.

-27 Cheng JH, She H, Han YP, Wang J, Xiong S, Asahina K, Tsukamoto H: Wnt antagonism inhibits hepatic stellate cell activation and liver fibrosis. Am J Physiol Gastrointest Liver Physiol 2008;294:G39-49.

28 Jiang F, Parsons CJ, Stefanovic B: Gene expression profile of quiescent and activated rat hepatic stellate cells implicates Wnt signaling pathway in activation. J Hepatol 2006;45:401-409.

29 Kweon SM, Chi F, Higashiyama R, Lai K, Tsukamoto H: Wnt Pathway Stabilizes MeCP2 Protein to Repress PPAR-gamma in Activation of Hepatic Stellate Cells. PLoS One 2016;11:e0156111.

-30 Li L, He L, Zhao JL, Xiao J, Liu M, Li X, Tang H: MiR-17-5p up-regulates YES1 to modulate the cell cycle progression and apoptosis in ovarian cancer cell lines. J Cell Biochem 2015;116:1050-1059.

-31 Jia J, Feng X, Xu W, Yang S, Zhang Q Liu X, Feng Y, Dai Z: MiR-17-5p modulates osteoblastic differentiation and cell proliferation by targeting SMAD7 in non-traumatic osteonecrosis. Exp Mol Med 2014;46:e107.

-32 Wu Q, Luo G, Yang Z, Zhu F, An Y, Shi Y, Fan D: miR-17-5p promotes proliferation by targeting SOCS6 in gastric cancer cells. FEBS Lett 2014;588:2055-2062.

-33 Corbett L, Mann J, Mann DA: Non-Canonical Wnt Predominates in Activated Rat Hepatic Stellate Cells, Influencing HSC Survival and Paracrine Stimulation of Kupffer Cells. PLoS One 2015;10:e0142794.

-34 Fatica A, Bozzoni I: Long non-coding RNAs: new players in cell differentiation and development. Nat Rev Genet 2014;15:7-21.

-35 Shi X, Sun M, Liu H, Yao Y, Song Y: Long non-coding RNAs: a new frontier in the study of human diseases. Cancer Lett 2013;339:159-166.

-36 Sarver AL, Subramanian S: Competing endogenous RNA database. Bioinformation 2012;8:731-733.

-37 Wang F, Ying HQ, He BS, Pan YQ Deng QW, Sun HL, Chen J, Liu X, Wang SK: Upregulated lncRNA-UCA1 contributes to progression of hepatocellular carcinoma through inhibition of miR-216b and activation of FGFR1/ERK signaling pathway. Oncotarget 2015;6:7899-7917.

-38 Wang J, Lei ZJ, Guo Y, Wang T, Qin ZY, Xiao HL, Fan LL, Chen DF, Bian XW, Liu J, Wang B: miRNA-regulated delivery of lincRNA-p21 suppresses beta-catenin signaling and tumorigenicity of colorectal cancer stem cells. Oncotarget 2015;6:37852-37870. 